\title{
Performance of Space-Frequency Codes in MIMO Channels with Frequency Offset
}

\author{
Dung Ngoc Dao and Chintha Tellambura \\ Electrical and Computer Engineering, University of Alberta \\ Edmonton, Alberta T6G 2V4, Canada \\ Email: \{dndung, chintha\}@ece.ualberta.ca \\ Telephone: (1-780) 492 7228, Fax: (1-780) 4921811
}

\begin{abstract}
We investigate the pair-wise error probability (PEP) performance of space-frequency (SF) codes over quasi-static, frequency-selective Rayleigh fading channels with frequency offset (FO). The PEP expression shows that the conventional $\mathrm{SF}$ code design criteria are remained valid. If the normalized FO (NFO) is less than 1\%, the performance loss is negligible. The loss increases with FO and with signal to noise ratio (SNR). We show that diversity can be used to mitigate the effects of FO. However, with large FO, the PEP is no longer exponentially decays with SNR. Therefore, we propose a SF coding scheme to combat effectively with even very high NFO $(20 \%)$. Simulation results confirm our theoretical evaluations.
\end{abstract}

\section{INTRODUCTION}

The space time (ST) codes proposed by Tarokh et al. [1] for coherent systems over quasi-static flat fading channels achieves the maximum diversity order (DO) $d=M N$, where $M$ and $N$ are the number of Tx (Tx) and $\mathrm{Rx}(\mathrm{Rx})$ antennas. The ST codes can achieve at least the designed DO in the FSF channels [1], but are not guaranteed to exploit all the available frequency diversity advantage of FSF channels. To overcome this, the authors in [2], [3], [4] and references therein propose new ST coding methods so that the maximum achievable DO is obtainable. It is found that in FSF channels, the maximum DO is $d=L M N$ where $L$ is the number of paths of channels. There are two approaches to design codes that can achieve this maximum DO [5]. In the first method, ST codes are constructed in the time domain, whereas in the second method called space-frequency (SF) coding, the source symbols are encoded across the subcarriers (or sub-carriers) of orthogonal frequency division multiplexing (OFDM) symbols in the frequency domain. Su et al. [6] derive SF code criteria which show an explicit relation between the SF code matrix and the characteristic parameters of FSF fading channels such as path delays and delay power distribution.

Like other error control coding methods, the performance of SF codes depends on the propagation environment [7]. Moreover, the performance of SF codes and OFDM may be affected by underlying factors such as frequency offset (FO), timing error, to name a few. A residual FO exists due to carrier synchronization mismatch and Doppler shift [8]. Residual FO breaks down the orthogonality among subcarriers, hence inter-carrier interference (ICI) is produced and bit error rate (BER) is increased consequently. Several papers have analyzed the effects of FO on the BER performance. For example in [9], BER is calculated for single input single output (SISO) OFDM systems with various modulation schemes. The authors in [10], [11] provide BER expressions of MIMOOFDM employing Alamouti's scheme [12]. However, to the best of our knowledge, the impact of FO on the SF code design criteria and the performance have not been investigated. This interesting question will be addressed in our paper.

We analytically show that the SF code design criteria still hold in the case of FO. The performance loss is negligible if the NFO is small. This loss is increased rapidly with the increase of NFO and with signal-to-noise ratio (SNR). When NFO is large, PEP performance of SF codes no longer decays exponentially. To mitigate the effect of $\mathrm{FO}$, we generalize the work of [13] for MIMO-OFDM with SF codes. The resulting SF codes not only achieve the same diversity and coding gains as the SF codes derived in [6] but also are capable of ICI self-cancellation. Simulations have showed that the ICI selfcancellation SF codes still yields good performance when the FO is extremely high, about $20 \%$.

The paper has seven sections. The model of MIMO-OFDM systems with FO is presented in Section II. Section III reviews the design criteria of SFC codes. In Section IV, we derive the PEP performance of SF codes in the presence of FO. In Section V, we propose a class of SF codes that reduce effectively FO effects to PEP. Section VI provides simulation results to support the theoretical analysis. We summarize the paper in Section VII.

\section{Frequency OfFSet in OFDM Systems}

This section briefly introduces the OFDM system with FO model to be analyzed for remaining parts of the paper.

\section{A. MIMO-OFDM System Model}

We consider the MIMO-OFDM system with $M$ Tx and $N \mathrm{Rx}$ antennas. The number of subcarriers in the OFDM modulator is $K$. The $L$-path quasi-static Rayleigh fading channel model is assumed identically for any link between Tx antenna $m(m=1, \ldots, M)$ and $\mathrm{Rx}$ antenna $n(n=1, \ldots, N)$. The channel impulse response in the time domain is [14]

$$
h_{m, n}(t, \tau)=\sum_{l=0}^{L-1} \alpha_{m, n}(t, l) \delta\left(\tau-\tau_{l}\right)
$$


where $\tau_{l}$ is the channel delay of the path $l$-th $(l=0, \ldots, L-$ $1), \delta($.$) denotes Dirac's delta function. The coefficients$ $\alpha_{m, n}(t, l)$ 's are complex channel gains of the path $l$ between Tx antenna $m$ and $\mathrm{Rx}$ antenna $n$, they are modeled as zero-mean complex Gaussian random variables with variance $E\left[\left|\alpha_{m, n}(l)\right|^{2}\right]=\delta_{l}^{2}$. Assume that coefficients $\alpha_{m, n}(t, l)$ are constant during an OFDM symbol duration, but vary randomly from symbol to symbol. Thus during a symbol period, the time index $t$ in (1) can be omitted. The total power of $L$-path channels is normalized, so that $\sum_{l=0}^{L-1} \delta_{l}^{2}=1$. The frequency response of the channel is

$$
H_{m, n}(f)=\sum_{l=0}^{L-1} \alpha_{m, n}(l) e^{-j 2 \pi f \tau_{l}}, \quad j=\sqrt{-1} .
$$

At the veceiver, assume that $\mathrm{Rx}$ subcarriers are perfectly sampled, let $c_{m}(k)$ is the subcarrier $k$-th $(k=0, \ldots, K-1)$ being sent from Tx antenna $m$, the received signal at the Rx antenna $n$ is $r_{n}(k)$

$$
r_{n}(k)=\sum_{m=1}^{M} c_{m}(k) H_{m, n}(k)+z_{n}(k)
$$

where $z_{n}(k)$ 's are independent noise samples, which are modelled as zero-mean complex white Gaussian variables. The Tx power from each antenna is normalized to 1 , resulting the noise variance per dimension is $M /(2 \rho)$ where $\rho$ is the average SNR at each Rx antenna.

\section{B. Model of MIMO-OFDM Systems with FO}

There is always a FO at the sampling points of received signal [15], [16]. In the SISO-OFDM system, the NFO $\varepsilon$ is defined by $\varepsilon=\delta f / \Delta f$, where $\delta f$ is the residual FO between the demodulated and transmitted subcarrier $k$-th, $\Delta f=1 / T_{s}$ is the subcarrier spacing, $T_{s}$ is the OFDM symbol duration. The NFO is assumed the same for all subcarriers of one OFDM symbol, but can vary from symbol to symbol. In SISO systems, the $k$-th received subcarrier is expressed as follows:

$$
r(k)=c(k) H(k) S(0)+I(k)+z(k)
$$

where $I(k)$ is the inter-carrier interference (ICI) from the other subcarriers to received subcarrier $k$; it is described by

$$
I(k)=\sum_{p=0}^{K-1} \sum_{p \neq k} c(p) H(p) S(p-k) .
$$

Coefficients $S(k)$ in (5) are expressed as:

$$
S(k)=\frac{\sin [\pi(k+\varepsilon)]}{K \sin \left[\frac{\pi}{K}(k+\varepsilon)\right]} \exp \left[j \pi\left(1-\frac{1}{K}\right)(k+\varepsilon)\right] \text {. }
$$

We generalize (4) for MIMO-OFDM systems. Let NFO of the transmission link from $\mathrm{Tx}$ antenna $m$ and $\mathrm{Rx}$ antenna $n$ is $\varepsilon_{m, n},(4)$ becomes:

$r_{n}(k)=\sum_{m=1}^{M} c_{m}(k) H_{m, n}(k) S_{m, n}(0)+\sum_{m=1}^{M} I_{m, n}(k)+z_{n}(k)$.

\section{SPACE-Frequency Code Design CRITERIA}

In the SF encoding process, the source data is twodimensional encoded across the space (over multiple antennas) and frequency (over the subcarriers of OFDM symbols). An SF codeword may occupy several OFDM symbols [4], [17] or within one OFDM symbol [3], [5], [6]. It is found that the maximal DO can be achieved by coding over the subcarriers of only one OFDM symbol [3], [6], whereas in [4], [17] the maximal diversity order is gained by coding over multiple OFDM symbols, that obviously causes higher coding and decoding delay. We adopt the approach in [6] for our analysis.

The input data symbols are divided into $b$-symbol source words then parsed into blocks and mapped to SF codewords. An SF codeword can be represented by a $K \times M$ matrix

$$
C=\left[\begin{array}{cccc}
c_{1}(0) & c_{2}(0) & \ldots & c_{M}(0) \\
c_{1}(1) & c_{2}(1) & \ldots & c_{M}(1) \\
\cdot & \cdot & . & \cdot \\
c_{1}(K-1) & c_{2}(\dot{K}-1) & \ldots & c_{M}(K-1)
\end{array}\right]
$$

At the receiver, the maximum likelihood (ML) decoder selects a codeword $E$ if its metric $M e$ is minimum

$$
M e=\sum_{k=0}^{K-1} \sum_{n=1}^{N}\left|r_{n}(k)-\sum_{m=1}^{M} e_{m}(k) H_{m, n}(k)\right|^{2} .
$$

Assume perfect channel state information (CSI) is available at the receiver but not at the Txter and perfect symbol timing, the PEP of a transmitted codeword $C$ and erroneously decoded codeword $E$ is upper bounded [6] as:

$$
P(C \rightarrow E) \leq\left(\begin{array}{c}
2 \Gamma N-1 \\
\Gamma N
\end{array}\right)\left(\prod_{m=1}^{\Gamma} \lambda_{i}\right)^{-N} \rho^{-\Gamma N} .
$$

In (10), $\Gamma$ is the rank of the matrix $Q=\Delta \circ R$, 。 denotes Hadamard product [18], $\lambda_{i}, i=1, \ldots, \Gamma$ are non-zero eigenvalues of $Q$,

$$
\Delta=(C-E)(C-E)^{\dagger}
$$

$R=R_{m, n}=E\left[H_{m, n} H_{m, n}^{\dagger}\right]=W \operatorname{diag}\left(\delta_{0}^{2}, \delta_{1}^{2}, \ldots, \delta_{L-1}^{2}\right) W^{\dagger}$,

where $R_{m, n}=E\left[H_{m, n} H_{m, n}^{\dagger}\right]$,

$$
\begin{gathered}
H_{m, n}=\left[H_{m, n}(0) H_{m, n}(1) \ldots H_{m, n}(K-1)\right]^{T}, \\
W=\left[\begin{array}{cccc}
1 & 1 & \ldots & 1 \\
w^{\tau_{0}} & w^{\tau_{1}} & \ldots & w^{\tau_{L-1}} \\
\cdot & \cdot & \ldots & . \\
w^{(K-1) \tau_{0}} & w^{(K-1) \tau_{1}} & \ldots & w^{(K-1) \tau_{L-1}}
\end{array}\right] .
\end{gathered}
$$

In (14), $w=e^{-j 2 \pi \Delta f}, \Delta f=1 / T_{s}$ is the subcarrier spacing, $T_{s}$ is the OFDM symbol duration. $X^{T}$ and $X^{\dagger}$ denote transpose and transpose conjugate of matrix $X$.

From (10), the DO of SF codes is $\Gamma N$, maximum achievable DO is equal $\min (L M N, K N)$ [6]. 


\section{Performance of SF Codes with Fo}

We continue the analysis with two assumptions below:

- AS1: Residual NFO's $\varepsilon_{m, n}$ are identical independent distributed (i.i.d) random variables. Their values are independent of the channel coefficients.

- AS2: The ICI terms $I_{m, n}(k)$ in (8) are independent.

In practice, AS2 is not completely true since there is a degree of correlation between transmitted streams. In OFDM systems, $K$ is typically 64 or larger. Therefore the central limit theorem can be applied to model the term $I_{m, n}(k)$ as a Gaussian random variable [16].

To investigate the PEP of SF codes with FO using formula (10), the channel coefficients $H_{m, n}^{S}(k)$ in (12) should be complex GRVs. This requirement can be met if $S_{m, n}(0)$ is deterministic or NFO is not a random variable (Case 1). In general case, $\varepsilon_{m, n}$ can be assumed to be independent and identically distributed random variables in the range $\left[E_{1}, E_{2}\right]$, their values can be changed from OFDM symbol to symbol (Case 2). However, the performance of SF codes with fixed values of FO is of greater interest since it provides a closer look at the performance of SF codes at specific FO values. For analytical tractability, we further make a third assumption:

- AS3: NFO $\varepsilon_{m, n}$ are constant and the same for all pair of indices $(m, n): \varepsilon_{m, n}=\varepsilon_{0}$.

Eq. (7) is rewritten with a slight modification. The channel coefficient and the $S_{m, n}(0)$ are grouped as

$r_{n}(k)=\sum_{m=1}^{M} c_{m}(k)\left[H_{m, n}(k) S_{m, n}(0)\right]+\sum_{m=1}^{M} I_{m, n}(k)+z_{n}(k)$.

Eq. (13) becomes

$$
H_{m, n}^{S}=S_{m, n}(0)\left[H_{m, n}(0) H_{m, n}(1) H_{m, n}(K-1)\right]^{T} .
$$

The correlation matrix in (12) has a new form

$$
\begin{gathered}
R_{m, n}^{S}=\bar{S}_{0}^{2} E\left[H_{m, n} H_{m, n}^{\dagger}\right], \\
R=R_{m, n}^{S}=\bar{S}_{0}^{2} R_{m, n} . \\
Q^{S}=\Delta \circ R_{m, n}^{S}=\sigma_{S}^{2}\left(\Delta \circ R_{m, n}\right)
\end{gathered}
$$

where $\bar{S}_{0}^{2}=E\left[\left|S_{m, n}(0)\right|^{2}\right]$. Note that the residual NFO is usually small, $\varepsilon \leq 0.2$ [16], the number of subcarriers $K \geq 8$, hence $K \sin (\pi \varepsilon / K) \approx \pi \varepsilon . \bar{S}_{0}^{2}$ can be evaluated as

$$
\bar{S}_{0}^{2}=E\left[\left|S_{m, n}(0)\right|^{2}\right]=\left[\operatorname{sinc}\left(\varepsilon_{0}\right)\right]^{2} .
$$

We can easily verify that: if the rank of matrix $Q$ is $\Gamma$, then $Q^{S}$ also has the same rank $\Gamma$; if $\lambda_{i}$ is an eigenvalue of $Q$ then $\lambda_{i}^{S}=\bar{S}_{0}^{2} \lambda_{i}$ is an eigenvalue of $Q^{S}$.
The first two moments of the term $I_{m, n}(k)$ in (14) by Gaussian approximation are calculated as follows.

$$
I_{m, n}(k)=\sum_{p=0}^{K-1} \sum_{p \neq n} c_{m}(p) H_{m, n}(p) S_{m, n}(p-k) .
$$

Assume that coded symbols $c_{m}(p)$ have zero-mean, then $E\left[I_{m, n}(k)\right]=0$. The variance $\sigma_{I_{m}}^{2}$ of $I_{m, n}(k)$ is evaluated in Appendix to be $\sigma_{I_{m}}^{2}=1-\bar{S}_{0}^{2}$. $\sigma_{I_{m}}^{2}$ is independent of indices $m$ and $n$, it is just dependent on the NFO through $\bar{S}_{0}^{2}$. In (15), let $Z_{n}(k)=I_{n}(k)+z_{n}(k)$ and $I_{n}(k)=\sum_{m=1}^{M} I_{m, n}(k)$. With $\mathrm{AS} 2, I_{n}(k)$ is a complex Gaussian random variable with zero-mean and variance $M\left(1-\bar{S}_{0}^{2}\right)$. Therefore $Z_{n}(k)$ is also a zero-mean complex Gaussian random variable with variance $\sigma_{Z}^{2}=M\left(1-\bar{S}_{0}^{2}+1 / \rho\right)$. Values of $\sigma_{Z}^{2}$ is identical for all Rx antennas. Hence equivalent SNR at each Rx antenna with $\mathrm{FO}$ is

$$
\rho^{S}=\frac{\bar{S}_{0}^{2}}{1-\bar{S}_{0}^{2}+1 / \rho} .
$$

We substitute $\lambda_{i}^{S}=\bar{S}_{0}^{2} \lambda_{i}$ and $\rho^{S}$ into (10), re-arrange the terms and get the PEP expression in the presence of FO as

$$
P(C \rightarrow E) \leq G\left(\begin{array}{c}
2 \Gamma N-1 \\
\Gamma N
\end{array}\right)\left(\prod_{i=1}^{\Gamma} \lambda_{i}\right)^{-N} \rho^{-\Gamma N}
$$

where

$$
G=\left(\frac{\bar{S}_{0}^{4}}{\rho\left(1-\bar{S}_{0}^{2}\right)+1}\right)^{-\Gamma N} .
$$

Comparing (10) and (23), we see that $G$ represents the PEP performance loss due to FO. By inspecting (20) and (24), we can draw following theoretical conclusions:

1) The design criteria for $\mathrm{SF}$ codes in the presence of FO is still valid for small values of FO.

2) At the same Tx power, the higher FO, the higher PEP performance loss.

3) Higher NFO, the further PEP curve shifts to the right. The shift of lower DO systems is larger than the shift of the system with higher DO.

4) If $\rho\left(1-\bar{S}_{0}^{2}\right) \gg 1$, at high Tx power

$$
\begin{aligned}
P(C \rightarrow E) \leq & \left(\begin{array}{c}
2 \Gamma N-1 \\
\Gamma N
\end{array}\right)\left(\prod_{m=1}^{\Gamma} \lambda_{i}\right)^{-N} \\
& \times\left(\frac{\bar{S}_{0}^{2}}{1-\bar{S}_{0}^{2}}\right)^{-\Gamma N} .
\end{aligned}
$$

So the PEP is no longer inversely proportional to SNR. The PEP reaches its floor level.

To complete this section, we note that one can derive the ML receiver using (7) and (9), the same result as (23) can be obtained. 


\section{ICI SELF-CANCELLATION WITH SF CODES}

$\mathrm{Su}$ et al. [6] show that if each row of the ST codeword is repeated $r$ times, the resulting SF code achieves at least DO $d=2 M N$. We consider $r=2$, a SF codeword is

$$
C_{1}=\left[\begin{array}{cccc}
c_{1}(0) & c_{2}(0) & \ldots & c_{M}(0) \\
c_{1}(0) & c_{2}(0) & \ldots & c_{M}(0) \\
\cdot & \cdot & \ldots & \dot{x} \\
c_{1}(K-1) & c_{2}(K-1) & \ldots & c_{M}(K-1) \\
c_{1}(K-1) & c_{2}(K-1) & \ldots & c_{M}(K-1)
\end{array}\right] .
$$

We develop the simple but effective idea of ICI selfcancellation of authors in [13] for MIMO-OFDM so that each row of ST code matrix is repeated once and the repeated row is sign reversed to form a new SF codeword.

$$
C_{2}=\left[\begin{array}{cccc}
c_{1}(0) & c_{2}(0) & \ldots & c_{M}(0) \\
-c_{1}(0) & -c_{2}(0) & \ldots & -c_{M}(0) \\
\cdot & \cdot & \ldots & \cdot \\
c_{1}(K-1) & c_{2}(K-1) & \ldots & c_{M}(K-1) \\
-c_{1}(K-1) & -c_{2}(K-1) & \ldots & -c_{M}(K-1)
\end{array}\right] .
$$

The number of OFDM subcarriers is $2 K$ in (26) and (27). The codeword formulation is different with the approach of [6], where each row is simply repeated $r$ times, $(r=2$ here). We can prove that the new mapping method yields the same coding gain and diversity gain (at least $d=2 M N$ ) compared with the approach in [6] for $r=2$, but our modified scheme integrates ICI self-cancellation capability. To prove that SF codes described in (26) and have the same coding gain and diversity gain, the reader can verify that if vector $X_{1}=\left[\begin{array}{lllllll}x_{1} & x_{2} & x_{3} & x_{4} & \ldots & x_{2 K-1} & x_{2 K}\end{array}\right]$ is an eigenvector of $Q_{1}=\Delta_{1} \circ R$, then $X_{2}=$ $\left[\begin{array}{lllllll}x_{1} & -x_{2} & x_{3} & -x_{4} & \ldots & x_{2 K-1} & -x_{2 K}\end{array}\right]$ is an eigenvector of $Q_{2}=\Delta_{2} \circ R$, where $Q_{1}, \Delta_{1}$ and $Q_{2}, \Delta_{2}$ are quantities associated with SF codewords defined in (10) and (11) respectively. Then if $\lambda$ is an eigenvalue associated with $X_{1}$, it is also an eigenvalue associated with $X_{2}$. Note that (10) provides an upper bound for PEP, the actual performance of different codes can be evaluated via simulations.

\section{Simulation Results AND Discussion}

The simulations are to verify the theoretical analysis in Section IV and V. We use a simple two-path channel with uniform power delay profile (delay time between the two paths is $5 \mu \mathrm{s}$ and COST207 six-path typical urban channel model [14]. In [6], each row of the space time (ST) code is repeated $r$ times $(1 \leq r \leq L)$ to form SF codewords. This approach reduces the spectrum efficiency. However the resulting SF codes are guaranteed to achieve diversity order of at least $d=r M N$. Note that coding gain and spectral efficiency are out of our interests. Thus we use algebraic ST convolutional codes [5], [19], codes rate $1 / 2$ with generator polynomial $(5,7)$ [20] without channel interleaver. The Viterbi decoder [19], [20] is used.

We compare the performance of SF codes for $1 \%, 10 \%$ and $20 \%$ NFO. Figs. 1 and 2 illustrate PEP curves of two OFDM systems with 64 subcarriers and two Tx antennas. System
1 is equipped with one Rx antenna and System 2 has two $\mathrm{Rx}$ antennas, so that the DO of the two systems is at least $d=2$ and $d=4$, respectively. Using (20), for $1 \%$ NFO, $S_{0}=0.9997, L_{0} \approx 1$ in the SNR region of interest $(\leq 30 \mathrm{~dB})$. Therefore, theoretically the performance loss is not significant. Figs. 1 and 2 confirm this conclusion. If the NFO is small, $1 \%$, the PEP curves almost overlap the PEP of the systems with no FO. In case NFO is $10 \%$, the PEP curves of all systems are shifted to the right, this shift is larger for the system with smaller DO. The SNR needed to compensate the effect of FO increases with NFO. However, when NFO is $10 \%$ the PEP curves are less steeper than the curves of PEP with $1 \%$ NFO. When NFO is $20 \%$, the PEP reduces slightly even SNR increases largely. The PEP performance reaches a floor at SNR is about $22 \mathrm{~dB}$. This symptom is more serious for low DO systems, where the floor level is higher than that of higher DO systems.

Fig. 3 presents the performance of the SF coding scheme proposed in [6] (SC1) and our modified scheme (SC2) with the same DO, $r=2, K=64, M=2, N=1$. SC2 with integrated ICI self-cancellation mechanism performs very well when $E_{0}=10 \%$. Performance of SC2 is always better than SC1. At $P E P=10^{-3}$, when $E_{0}=0$ and $10 \%$, SC2 provides $0.5 \mathrm{~dB}$ and $1.5 \mathrm{~dB}$ improvement respectively. More important, SC2 lowers the error floor level extensively even at high FO $E_{0}=20$.

\section{CONCLUSION}

We analyzed PEP performance of SF codes with FO. Through the PEP upper bound expression of SF codes with FO, we showed that the code design criteria remained valid. The effect of FO is more severe for low DO SF codes. To minimize the effects of FO, we proposed an enhanced ICI self-cancellation scheme using SF codes. Finally we conclude that diversity not only improves the performance of OFDM systems in the dispersive channels, but also makes the system robust to the effects of FO.

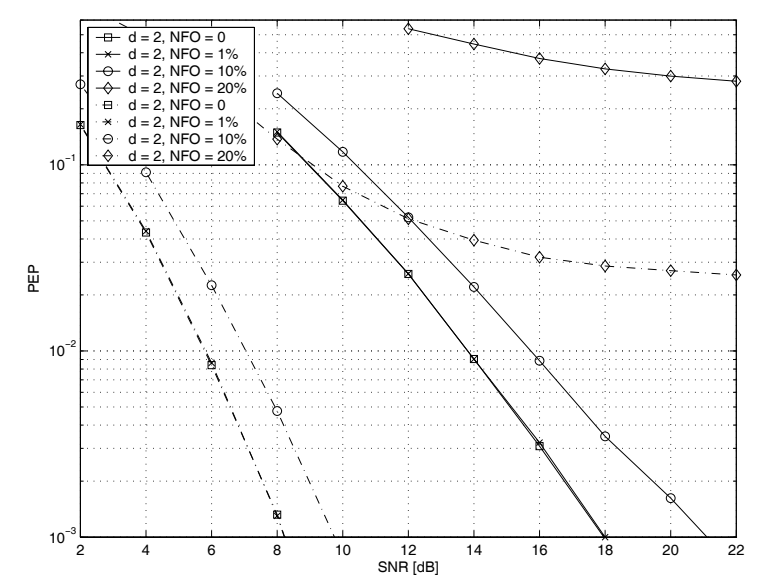

Fig. 1. Performance SF codes with $K=64$, constant FO for the two-path channel model. 


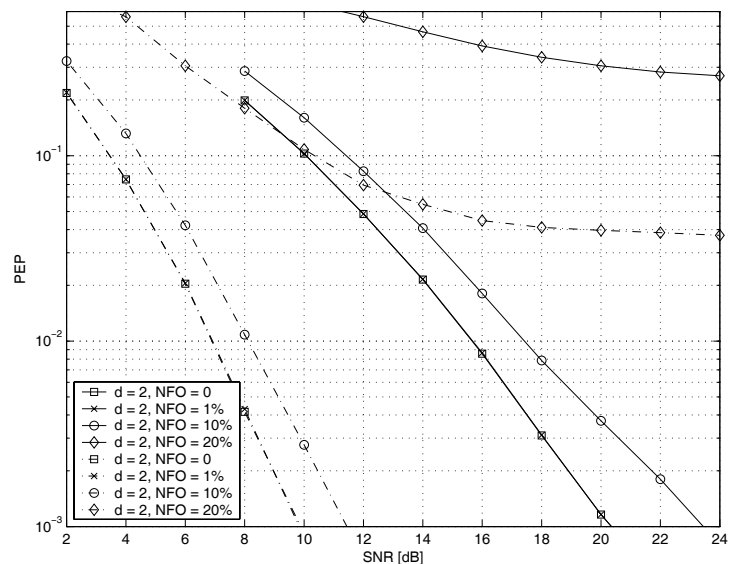

Fig. 2. Performance of SF codes with $K=64$, constant FO for the six-path COST207 typical urban channel model.

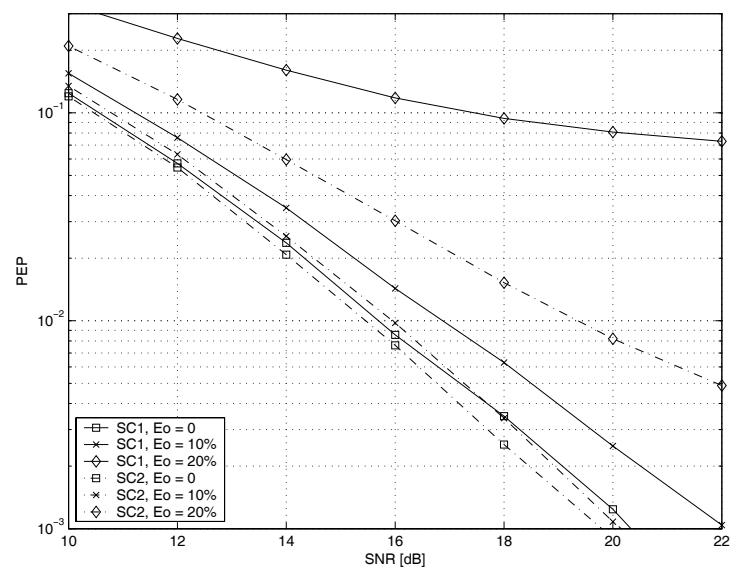

Fig. 3. Performance SF codes with $K=128, r=2$, two-path channel model with and without ICI self-cancellation

\section{APPENDIX}

We calculate $\sigma_{I_{m}}^{2}$ as follows:

$$
\begin{aligned}
\sigma_{I_{m}}^{2}= & E\left[\left|I_{m}(k)\right|^{2}\right] \\
= & E\left[\left|\left(\sum_{p=0}^{K-1} c_{m}(p) H_{m, n}(p) S_{m, n}(p-k)\right)\right|^{2}\right] \\
& -E\left[\left|c_{m}(k) H_{m, n}(k) S_{m, n}(0)\right|^{2}\right] \\
= & \sum_{p=0}^{K-1} E\left[\left|c_{m}(p)\right|^{2}\right] E\left[\left|H_{m, n}(p)\right|^{2}\right] E\left[\left|S_{m, n}(p-n)\right|^{2}\right] \\
& -E\left[\left|c_{m}(k)\right|^{2}\right] E\left[\left|H_{m, n}(k)\right|^{2}\right] E\left[\left|S_{m, n}(0)\right|^{2}\right]
\end{aligned}
$$

In (28), the term $E\left[\left|c_{m}(k)\right|^{2}\right]$ is the signal power, it is normalized to 1 . The second term is the average of the channel power, and it is also normalized to $1,(27)$ becomes

$$
\sigma_{I_{m}}^{2}=\sum_{p=0}^{K-1} E\left[|S(p-k)|^{2}\right]-\bar{S}_{0}^{2}
$$

It is found in [8] that the sum $\sum_{p=0}^{K-1} E\left[|S(p-k)|^{2}\right]=1$.

Substitute the sum into (28) completes the proof.

\section{REFERENCES}

[1] V. Tarokh, N. Seshadri and A. R. Calderbank, "Space-time codes for high data rate wireless communication: Performance analysis and code construction," IEEE Trans. Inform. Theory., vol. 44, pp. 744-765, Mar. 1998.

[2] M. Qin and R. Blum, "Properties of space-time codes for frequencyselective channels," IEEE Trans. Signal Processing, vol. 52, pp. $694-$ 702, Mar. 2004.

[3] H. Bolcskei and A. J. Paulraj, "Space-frequency coded broadband OFDM systems," in Proc. IEEE Wireless Communications Networking Conf. (WCNC), vol. 1, Sep. 2000, pp. $1-6$.

[4] Z. Liu, Y. Xin and G.B. Giannakis, "Space-time-frequency coded OFDM over frequency-selective fading channels," IEEE Trans. Signal Processing, vol. 50, pp. 2465 - 2476, Oct. 2002.

[5] H. El Gamal, A. R. Hammons, Jr., Y. Liu, M. P. Fitz and O. Y. Takeshita, "On the design of space-time and space-frequency codes for MIMO frequency-selective fading channels," IEEE Trans. Inform. Theory., vol. 49, pp. 2277 - 2292, Sept. 2003.

[6] W. Su, Z. Safar, M. Olfat and K. J. R. Liu, "Obtaining full-diversity space-frequency codes from space-time codes via mapping," IEEE Trans. Signal Processing, vol. 51, pp. 2905 - 2916, Nov. 2003.

[7] H. Bolcskei, M. Borgmann and A. J. Paulraj, "Impact of the propagation environment on the performance of space-frequency coded MIMOOFDM," IEEE J. Select. Areas. Commun., vol. 21, pp. 427 - 439, April 2003.

[8] T. Pollet, M. V. Bladel and M. Moeneclaey, "BER sensitivity of OFDM systems to carrier frequency offset and wiener phase noise," IEEE Trans. Commun., vol. 43, no. 2/3/4, pp. 191-193, Feb./March/April 1995.

[9] K. Sathananthan and C. Tellambura, "Probability of error calculation of OFDM systems with frequency offset," IEEE Trans. Commun., vol. 49, pp. $1884-1888$, Nov. 2001.

[10] H.-K. Song, S.-J. Kang, M.-J. Kim and Y.-H. You, "Error performance analysis of STBC-OFDM systems with parameter imbalances," IEEE Trans. Broadcast., vol. 50, pp. 76 - 82, Mar. 2004.

[11] R. Narasimhan, "Performance of diversity schemes for OFDM systems with frequency offset, phase noise, and channel estimation errors," IEEE Trans. Commun., vol. 50, pp. 1561 - 1565, Oct. 2002.

[12] S. Alamouti, "A simple transmit diversity technique for wireless communications," IEEE J. Select. Areas. Commun., vol. 16, pp. 1451 - 1458, Oct. 1998.

[13] Y. Zhao and S. Häggman, "Intercarrier interference self-cancellation scheme for OFDM mobile communication systems," IEEE Trans. Commun., vol. 49, pp. 1185-1191, July 2001.

[14] G. L. Stuber, Principles of mobile communications, 2nd ed. Norwell, MA: Kluwer, 2001.

[15] P. Moose, "A technique for orthogonal frequency division multiplexing frequency offset correction," IEEE Trans. Commun., vol. 42, no. 10, pp. 2908-2914, Oct. 1994.

[16] T. Keller and L. Hanzo, "Adaptive multicarrier modulation: a convenient framework for time-frequency processing in wireless communications," IEEE Proc., vol. 88, pp. 611 - 640, May 2000.

[17] Yi Gong and K. B. Letaief, "An efficient space-frequency coded OFDM system for broadband wireless communications," IEEE Trans. Commun., vol. 51, pp. 2019 - 2029, Dec. 2003.

[18] R. A. Horn and C. R. Johnson, Matrix Analysis. Cambridge, U.K.: Cambridge Univ. Press, 1985.

[19] A. R. Hammons, Jr., and H. El Gamal, "On the theory of space-time codes for PSK modulation," IEEE Trans. Inform. Theory., vol. 46, pp. 524 - 542, March 2000.

[20] J. G. Proakis, Digital Communications, 4th ed. New York: McGrawHill, 2001. 